\title{
PRESENTACIÓN DEL DOCUMENTO TÉCNICO “ANÁLISIS DE LA SITUACIÓN DEL CÁNCER EN EL PERÚ, 2018”
}

\author{
PRESENTATION OF THE TECHNICAL DOCUMENT “ANALYSIS OF THE SITUATION OF CANCER IN PERU, 2018”
}

\author{
Willy Ramos', Jhony A. De La Cruz-Vargas²
}

El diseñoy planificación de las intervenciones de prevención y control así como la evaluación de las intervenciones ya implementadas requieren un análisis situacional a partir de diversos sistemas y fuentes de información que permitan dirigir y priorizar los recursos hacia las poblaciones más vulnerables y/o desfavorecidas. Los análisis de situación de salud constituyen una herramienta poderosa para la identificación de necesidades y prioridades siendo esenciales para la construcción de políticas en salud. ${ }^{(1,2)}$

El 27 de diciembre de 2019, el Centro Nacional de Epidemiología, Prevención y Control de Enfermedades presentó en el Paraninfo del Ministerio de Salud el “Análisis de la situación del cáncer en el Perú, 2018" (3). Dicho documento técnico busca contribuir en base al análisis de datos de diversas fuentes de información al diseño de las intervenciones de prevención y control del cáncer en el país, particularmente en la formulación del Plan Nacional de Cuidados Integrales del Cáncer 2020-2024 que representa la continuación del Plan Esperanza(4).

El "Análisis de la situación del cáncer en el Perú, 2018" muestra que la incidencia y mortalidad del cáncer en el Perú se explica en gran medida por los determinantes sociales de la salud (pobreza, educación) y la exposición a factores de riesgo. Los datos de la Encuesta Nacional de Hogares muestran que la pobreza en el Perú en los últimos 11 años presenta una tendencia descendente, pasando de 32,0\% en 2007 a 17,4\% en 2017. El promedio de años de estudios de la población nacional en 2017 fue de 10,3 años en comparación con 2007 en que el promedio fue de 9,9 años, lo cual indica que una fracción importante de la población no completó educación secundaria.

Las Encuestas Nacionales de Prevención y Consumo de Drogas en población general ylas Encuestas Demográficas y de Salud Familiar 2014-2017 muestran que el consumo de tabaco y alcohol tienen una tendencia descendente, la obesidad y el sobrepeso tienen tendencia ascendente; mientras que, el bajo consumo de frutas y verduras presenta tendencia estacionaria. Las infecciones oncogénicas muestran prevalencias variables en población general y poblaciones vulnerables siendo las más prevalentes la infección por Helicobacter pylori y la infección por el virus del papiloma humano (VPH).

El documento técnico presentado muestra que para el año 2018, la Agencia Internacional para la Investigación del Cáncer estima que la tasa estandarizada de incidencia de cáncer en el Perú era de 192,6 casos nuevos por 100000 habitantes lo que representa, el diagnóstico de 66627 casos nuevos en una población de 32551811 personas para ese año ${ }^{(5)}$. Asimismo, a partir del Registro de Defunciones, se estima que en 2016 se produjeron 32163 muertes por cáncer lo que representa una tasa ajustada de mortalidad de 122,9 defunciones por cada 100000 habitantes. Las neoplasias malignas constituyeron la segunda causa de mortalidad global y los departamentos con mayor tasa ajustada de mortalidad fueron Huánuco, Junín, Apurímac, Ayacucho y San Martín. Los cánceres con mayor mortalidad fueron los de próstata, estómago, cérvix, hígado y vías biliares y tráquea bronquios y pulmón.

Con relación a la respuesta del estado y de los servicios de salud frente al cáncer, el "Análisis de la situación del cáncer en el Perú, 2018" encuentra que existió un incremento del número de especialistas en oncología clínica y radioterapia con relación al primer análisis de la situación del cáncer publicado en $2013^{(6)}$; sin embargo, la

'Centro Nacional de Epidemiología, Prevención y Control de Enfermedades, Ministerio de Salud. Lima, Perú.

${ }^{2}$ Instituto de Investigación en Ciencias Biomédicas, Facultad de Medicina Humana, Universidad Ricardo Palma. Lima, Perú.

Citar como: Willy Ramos, Jhony A. De La Cruz-Vargas. Presentación del documento técnico "Análisis de la situación del cáncer en el Perú, 2018". Rev Rev. Fac. Med. Hum. Enero 2020; 20(1):10-11. DOI 10.25176/RFMH.v20i1.2704

Journal home page: http://revistas.urp.edu.pe/index.php/RFMH

Artículo publicado por la Revista de la Facultad de Medicina Humana de la Universidad Ricardo Palma. Es un artículo de acceso abierto, distribuído bajo los términos de la Licencia Creative Commons: Creative Commons Attribution 4.0 International, CC BY 4.0 (https://creativecommons.org/licenses/by/4.0/), que permite el uso no comercial, distribución y reproducción en cualquier medio, siempre que la obra original sea debidamente citada. Para uso comercial, por favor póngase en contacto con revista.medicina@urp.pe 
inequidad en su distribución no se ha reducido. Para el año 2017, de los 204 especialistas en oncología clínica censados, el 73,0\% se concentraban en Lima y Callao. En 2018, se disponía a nivel nacional de 69 especialistas en radioterapia los cuales se concentraban también en Lima y Callao (82,6\%). Similar situación ocurrió con los cirujanos oncólogos observándose que de 178 especialistas censados en 2017, el 79,2\% se encontraban en Lima y Callao.

En 2017 a nivel nacional se disponía de 110 equipos de mamografíaoperativosysibientodoslos departamentos disponían de al menos un equipo, en departamentos como Cajamarca, Cusco, Piura, Lima, Loreto, Ica, La Libertad y Puno el número de equipos era insuficiente para la población objetivo. Todos los departamentos contaban con al menos un servicio de anatomía patológica con excepción de los departamentos de Amazonas, Apurímac, Huánuco, Madre de Dios, Pasco y Tumbes. Los servicios con mayor concentración en establecimientos públicos fueron los de radioterapia y trasplante de médula ósea, radioterapia se encontraba restringido a Lima, Arequipa, La Libertad, Cusco y Junín; mientras que, el trasplante de médula ósea a Lima, Lambayeque y Arequipa.

El análisis de vulnerabilidad territorial incluido en el "Análisis de la situación del cáncer en el Perú, 2018" muestra que los departamentos más vulnerables para la respuesta de prevención y control del cáncer fueron Huánuco, Pasco, Ayacucho, Amazonas, Cajamarca, Loreto y San Martín. Estos departamentos requieren de intervención prioritaria en el corto plazo para el fortalecimiento de las intervenciones de prevención y control del cáncer. Los departamentos de Apurímac, Ica, Huancavelica, Madre de Dios, Junín, Lambayeque y Piura, se encontraron también en condición de vulnerabilidad por lo que requieren intervención en el corto-mediano plazo.

Se espera que la información presentada en el documento técnico "Análisis de la situación del cáncer en el Perú, 2018" contribuya a optimizar las intervenciones de prevención y control implementadas por el estado peruano (preventivas, de tamizaje y diagnóstico temprano, de acceso y atención de los casos así como de cuidados paliativos), particularmente en los departamentos más vulnerables con fines de mejorar la efectividad y eficiencia de las intervenciones así como para llevarlas a los grupos de mayor riesgo y/o exclusión. Se espera que este documento técnico contribuya también a la discusión de intervenciones basadas en la evidencia que podrían implementarse en el país no solo por parte de las instituciones del estado sino también de la Academia y la sociedad civil.

\section{REFERENCIAS BIBLIOGRÁFICAS}

1. Valdez Huarcaya W, Berto Gonzáles M, Oyola-García AE, Vidal Valenzuela L, Vílchez Gutarra A, Ormaeche Macassi M. Análisis de situación de salud del Perú. Lima: Centro Nacional de Epidemiología, Prevención y Control de Enfermedades; 2017.

2. Ramos W, Guerrero N, Medina J, Guerrero PC. Análisis de la situación del cáncer en el Perú, 2018. Lima: Centro Nacional de Epidemiología, Prevención y Control de Enfermedades; 2019.

3. Escobar-Díaz FA, Castillo-Santana PT, Rodríguez-Salazar N, QuinteroCabezas CJ, Castañeda-Orjuela CA. Experiencias departamentales en la construcción, divulgación y uso del análisis de situación de salud, Colombia 2016. Rev. Salud Pública. 2017;19(3):368-73.
4. Plan Nacional para la Atención Integral del Cáncer y Mejoramiento del Acceso a los Servicios Oncológicos en el Perú "Plan Esperanza". Decreto Supremo N 009-2012-SA. Diario el Peruano: Normas Legales, № 477902, (03/11/2012).

5. International Agency for Reasearch on Cancer (IARC). Cancer Today. Lyon; IARC; 2018. Disponible en: https://gco.iarc.fr/today/home.

6. Ramos W, Venegas D, Medina J, Guerrero PC, Cruz A. Análisis de la situación del cáncer en el Perú, 2013. Lima: Dirección General de Epidemiología; 2013. 